\title{
Switched Interleaving Techniques with Limited Feedback for Interference Mitigation in DS-CDMA Systems
}

\author{
Yunlong Cai, Rodrigo C. de Lamare, and Rui Fa
}

\begin{abstract}
In this paper we propose a novel switchedinterleaving algorithm based on limited feedback for both uplink and downlink DS-CDMA systems. The proposed switched chipinterleaving DS-CDMA scheme requires the cooperation between the transmitter and the receiver, and a feedback channel sending the index of the interleaver to be used. The transmit chipinterleaver is chosen by the receiver from a codebook of interleaving matrices known to both the receiver and the transmitter and the codebook index is sent back using a limited number of bits. In order to design the codebook, we consider a number of different chip patterns by using random interleavers, block interleavers and a proposed frequently selected patterns method (FSP). The best interleaving patterns are chosen by the selection functions of the received signal to interference plus noise ratio (SINR) for both downlink and uplink systems. Since the selection function needs to determine the best interleaver based on the channel state information, it is necessary to predict reliably the channel state information for typical delay values. We present symbol-based and block-based linear minimum mean squared error (MMSE) receivers for interference suppression. Simulation results show that our proposed algorithm achieves significantly better performance than the conventional DS-CDMA (C-CDMA) systems and the existing chip-interleaving, linear precoding and adaptive spreading techniques.
\end{abstract}

Index Terms-Direct sequence code division multiple access (DS-CDMA), interference suppression, multiuser detection, limited feedback, chip-interleaving.

\section{INTRODUCTION}

$\mathbf{T}$ EMPORAL variations in multipath fading channel restricts the performance of direct-sequence code division multiple access (DS-CDMA) systems. A typical fade in a wireless channel lasts over several bits duration and causes burst errors. This is typically mitigated by bit interleaving [1]. Recently, a number of works have considered chip-interleaving for CDMA systems. The work in [2] demonstrates that chipinterleaving overperforms bit-interleaving. This result is based on the assumption that the receiver has perfect channel state

Paper approved by X. Wang, the Editor for Multiuser Detection and Equalization of the IEEE Communications Society. Manuscript received February 25, 2009; revised July 8, 2009, December 1, 2009, April 23, 2010, and September 8, 2010.

Part of this manuscript was presented at ICC '09 and WCNC '09.

Y. Cai is with the Department of Information Science and Electronic Engineering, Zhejiang University, Hangzhou 310027, China (e-mail: ylcai@zju.edu.cn).

R. C. de Lamare is with the Communications Research Group, Department of Electronics, University of York, YO10 5DD York, U.K. (e-mail: rcd1500@ohm.york.ac.uk).

$\mathrm{R}$. Fa is with the Department of Electrical Engineering and Electronics, University of Liverpool, L69 3GJ, U.K. (e-mail: r.fa@liverpool.ac.uk).

Digital Object Identifier 10.1109/TCOMM.2011.051711.090120A information. In [3] the authors proposed a pilot-aided chipinterleaving algorithm for the uplink of a CDMA system, and the proposed system is capable of outperforming the conventional DS-CDMA (C-CDMA) one by using the estimated channel state information. The work in [28] compared and analyzed two fade-resistant transmission systems, which are chip-interleaved and parallel transmission systems. A CDMA system with partitioned chip-interleaving algorithm has been proposed by Schlegel in [9], where the signature sequences are partitioned into sections, which are interleaved before transmission, and processed at the receiver. Zhou et al. [8] proposed a multiuser-interference (MUI) free transceiver for the frequency-selective multipath channels, which can be viewed as block-spreading chip-interleaving algorithm.

Channel adaptive techniques [10]-[13] are expected to be exploited in the next generation of wireless communications systems. These signaling approaches allow the transmitter to adapt to the propagation conditions [14]. This implies that the transmitter requires some form of knowledge of the channel state information at the transmitter. While it is sometimes possible to use the uplink channel estimate for the downlink in time division duplexing (TDD) systems, a problem arises when it is implemented with frequency division duplexing (FDD) systems, which lack downlink and uplink channel reciprocity. Alternatively, the transmitter can obtain the channel knowledge by allowing the receiver to send a small number of feedback bits. Then, it can use the feedback information to modify the transmit signal prior to transmission over the channel. Limited feedback approaches have been widely investigated in multiple-input multiple-output (MIMO) systems [15]-[22]. In particular, the works in [16] and [17] introduced feedback information corresponding to beamforming, and limited feedback precoding was proposed in [15] and [18]. However, prior work [19]-[22] has focused on the quantization of the channel information, which encounters problems in time-varying fading and requires a significant amount of bits for satisfactory performance. A number of works on adaptive spreading techniques for DS-CDMA systems have been discussed in [29]-[33]. An optimization algorithm without multipath, based on individual updates, has been presented and analyzed in [29]-[31]. In [32], joint transmitter-receiver adaptation is studied for the uplink of a system with short signature sequences, and two alternating update algorithms are derived for estimating the transmitter coefficients in the presence of multipath. The work in [33] studied the performance of the signature optimization with limited feedback using a random vector quantization (RVQ) scheme. 


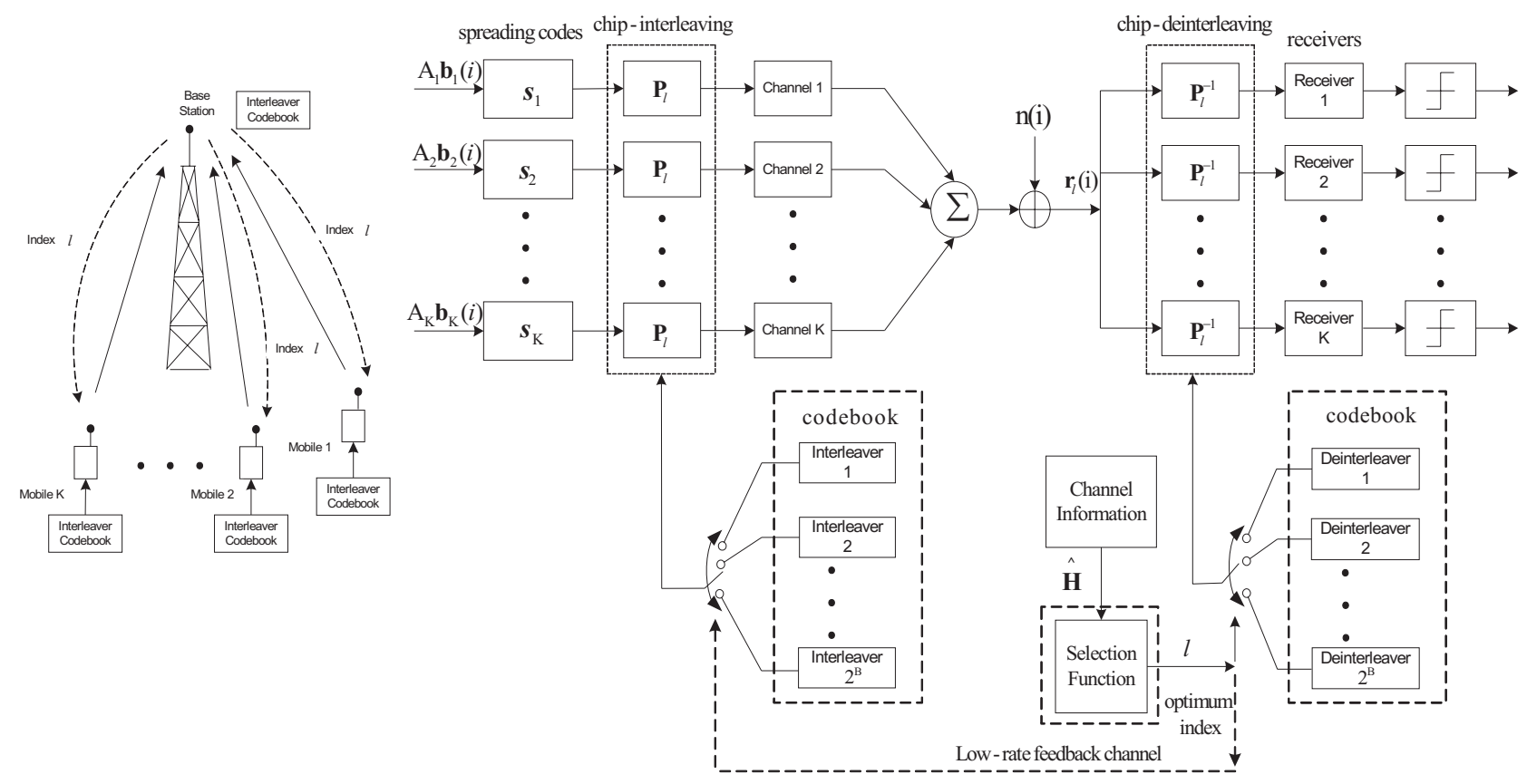

Fig. 1. Proposed uplink limited feedback-based SIDS-CDMA model and transceiver structure.

In this paper, we investigate a novel chip-interleaving algorithm based on limited feedback. A set of possible chipinterleavers are constructed and pre-stored at both the transmitter and receiver. During the transmission, the optimum interleaver is chosen by the selection function at the receiver, which then relays the index of the interleaver to the transmitter by a low-rate feedback channel or link. The transmitter will send data by using the interleaver corresponding to the index sent from the receiver in this particular channel situation. It is worth to note that the optimum interleaver may change per transmission block. In order to design the codebook, we consider a number of different chip patterns by using random interleavers, block interleavers [3] and a proposed frequently selected patterns method (FSP). Since the selection function needs to determine the best interleaver based on the channel state information, it is necessary to predict reliably the channel state information for typical delay values. The symbol-based and block-based minimum mean squared error (MMSE) receivers for the proposed scheme are investigated. The proposed scheme shows substantial performance gains, has much lower requirements for feedback bits than most channel-based feedback schemes, and also has increased robustness against channel variations. The simulations show that our proposed algorithm achieves better performance than the C-CDMA systems and the existing chip-interleaving, linear precoding and adaptive spreading schemes. The main contributions of this paper are:

I) Novel limited-feedback techniques combined with chipinterleaving algorithms are introduced for interference suppression in uplink and downlink DS-CDMA systems.

II) We develop symbol-by-symbol and block linear MMSE receivers for the proposed scheme.

III) We design the selection functions based on the different MMSE receivers.
IV) Several chip-interleaving codebook design methods are proposed.

The paper is structured as follows. Section II briefly describes the proposed switched interleaving DS-CDMA (SIDSCDMA) system models and the relevant MMSE receivers. Section III presents the selection functions for both uplink and downlink. Techniques to design chip-interleaver codebooks are described in Section IV. The simulation results are presented in Section V. Section VI draws the conclusions.

\section{Proposed System Models ANd MMSE Receivers}

In this section, we focus on the description of the proposed system models and linear MMSE receivers. Other receivers are possible such as decision feedback [34] and [35] and successive and parallel interference cancellation [36]. We first consider an uplink scenario and describe the proposed SIDSCDMA system model, where the channel coefficients vary per symbol. Then we explain how the uplink scenario can be extended to the case of the downlink. Subsequently, we introduce the design of the relevant linear block and symbolbased MMSE receivers.

\section{A. System Model}

The proposed uplink limited feedback-based SIDS-CDMA model and transceiver structure is presented in Fig. 1. All the mobile stations and the base station are equipped with the same codebook of chip-interleavers. We assume that the channel varies per symbol duration, and the base station predicts the future uplink channel coefficients with one block length for each user [4]-[6]. Based on the interleaving index patterns and the predicted channel information, the selection function at the receiver selects an index from the codebook. This index corresponds to the optimum interleaver, and is updated per block. With the aid of a low-rate feedback 
channel, the base station relays the index to each mobile station. The signal is transmitted after preprocessing by a chipinterleaver, which is chosen from the codebook available at the transmitter. The received vector is first processed by a relevant chip-deinterleaver and then handled by linear receivers.

Let us consider the uplink of an uncoded synchronous binary phase-shift keying (BPSK) DS-CDMA system with $K$ users, $N$ chips per symbol and $M$ symbols per block. We assume that the proposed algorithm is employed in the multipath fading channels with $L_{p}$ propagation paths, the delays are multiples of the chip duration and the receiver is synchronized with the main path. The channel vector of the $\beta$-th symbol in the $i$-th block for the $k$-th user is defined as $\mathbf{h}_{k}^{(\beta)}(i)=\left[h_{0}^{(\beta)}(i), \ldots, h_{L_{p}-1}^{(\beta)}(i)\right]^{T}$, where $\beta=1 \ldots M$, and $(.)^{T}$ denotes transpose. The $M N$-dimensional received vector of the $i$-th block is given by

$$
\mathbf{r}_{l}(i)=\sum_{k=1}^{K} A_{k} \mathbf{P}_{l}^{-1} \breve{\mathbf{H}}_{k}(i) \mathbf{P}_{l} \mathbf{S}_{k} \mathbf{b}_{k}(i)+\mathbf{n}(i),
$$

where $\mathbf{b}_{k}(i)=\left[b_{1}^{(k)}(i), b_{2}^{(k)}(i), \ldots, b_{M}^{(k)}(i)\right]^{T}$, denotes the $i$-th block of symbols for user $k, b_{m}^{(k)}(i) \in\{ \pm 1\}, m=1 \ldots M$. The quantity $A_{k}$ is the amplitude associated with user $k$. The matrices $\mathbf{P}_{l}$ and $\mathbf{P}_{l}^{-1}$ denote the $l$-th $M N \times M N$ interleaving and relevant deinterleaving matrices, respectively. $\mathbf{P}_{l}$ and $\mathbf{P}_{l}^{-1}$ are designed by the interleaving patterns of the codebook, where $l=1 \ldots 2^{B}, B$ is the number of feedback bits, the number $2^{B}$ denotes the length of the interleaving codebook. The function of this interleaving matrix is to permute the orders of these chips per block. The quantity $\mathbf{S}_{k}=\mathbf{s}_{k} \otimes \mathbf{I}_{M}$ is the $M N \times M$ spreading code matrix, where we define $\mathbf{s}_{k}=\left[a_{k, 1}, \ldots, a_{k, N}\right]^{T}$ as the $N \times 1$ signature sequence vector for user $k$. The signature $\mathbf{s}_{k}$ is repeated from symbol to symbol, and $a_{k, \gamma} \in\{ \pm 1 / \sqrt{N}\}, \gamma=1 \ldots N$. The quantity $\mathbf{I}_{M}$ represents an $M \times M$ identity matrix, and $\otimes$ is the Kronecker product. The $M N \times M N$ matrix $\breve{\mathbf{H}}_{k}(i)$ is generated by discarding the last $L_{p}-1$ rows of the matrix $\mathbf{H}_{k}(i)$, which represents the $\left(M N+L_{p}-1\right) \times M N$ Toeplitz channel matrix of the $k$-th user. The matrix $\mathbf{H}_{k}(i)$ has the structure shown in (2), whose columns are shifted by one position versions of the $M$ channel vectors $\mathbf{h}_{k}^{(\beta)}(i)$ in the $i$-th block. The vector $\mathbf{n}(i)=\left[n_{1}(i), \ldots, n_{M N}(i)\right]^{T}$ is the complex Gaussian noise vector, $E\left[\mathbf{n}(i) \mathbf{n}^{H}(i)\right]=\sigma^{2} \mathbf{I}_{M N}$, the quantity $\sigma^{2}$ denotes the noise variance. $(.)^{H}$ denotes Hermitian transpose. $E[$.$] stands$ for ensemble average.

We note that the proposed downlink scheme has the same system model, but the channel matrices only corresponds to the desired user since the composite signals broadcasted from the base station to the particular user experience the same propagation conditions. In this case, the base station broadcasts signals by employing the interleaver entry corresponding to the feedback index of the user of interest, and each user is deinterleaved using the same entry in the codebook. The proposed downlink system provides the best performance for the user of interest among the codebook entries. Note that the index selected in the downlink is the optimum index for the desired user. In the following section, the proposed linear MMSE receivers are described.

\section{B. Proposed MMSE receivers}

In this part, we present the design of the linear receivers of our proposed scheme based on the MMSE criterion [23], which are symbol-based and block-based receivers.

1) MMSE Symbol-based Receiver: The symbol-based MMSE detector is designed as a parameter vector which operates with a symbol-length received data. Let us recall the proposed system model in (1) with the $M N \times 1$ received vector $\mathbf{r}_{l}(i)$. We assume that the vector $\mathbf{r}_{l}(i)=\left[\mathbf{r}_{l, 1}^{\prime T}(i), \mathbf{r}_{l, 2}^{\prime T}(i), \ldots, \mathbf{r}_{l, M}^{\prime T}(i)\right]^{T}, \mathbf{n}(i)=$ $\left[\breve{\mathbf{n}}_{1}^{T}(i), \breve{\mathbf{n}}_{2}^{T}(i), \ldots, \breve{\mathbf{n}}_{M}^{T}(i)\right]^{T}$, where $\mathbf{r}_{l, j}^{\prime}(i)$ and $\breve{\mathbf{n}}_{j}(i)$ are $N \times$ 1 vectors. We define the matrix $\mathbf{\Theta}_{l}^{(k)}=\mathbf{P}_{l}^{-1} \breve{\mathbf{H}}_{k}(i) \mathbf{P}_{l}$, which is partitioned into $N \times N$ submatrices as $\mathbf{T}_{j, m}^{(k, l)}=$ $\Theta_{l}^{(k)}((j-1) N+1: j N,(m-1) N+1: m N)$, where $j=1 \ldots M$ and $m=1 \ldots M$. The operation generates a submatrix by taking row $(j-1) N+1$ to row $j N$ and column $(m-1) N+1$ to $m N$ from the matrix $\Theta_{l}^{(k)}$. Thus, we obtain the $j$-th received symbol per block of user $k$

$$
\mathbf{r}_{l, j}^{\prime}(i)=\sum_{k=1}^{K} \sum_{m=1}^{M} A_{k} \overline{\mathbf{s}}_{l, j, m}^{(k)}(i) b_{m}^{(k)}(i)+\breve{\mathbf{n}}_{j}(i),
$$

where $N \times 1$ vector $\overline{\mathbf{s}}_{l, j, m}^{(k)}(i)=\mathbf{T}_{j, m}^{(k, l)} \mathbf{s}_{k}$. The received symbol vector $\mathbf{r}_{l, j}^{\prime}(i)$ consists of $M K$ different signals corresponding to the different users and symbols per block. Thus, we can design a group of symbol-based receivers $\mathbf{w}_{l, j, m}^{(k)}(i)$ according to the $M K$ different signals, where $k=1 \ldots K$. Let us consider the mean square error (MSE) cost function of the $j$-th received symbol per block for user $k$ of the branch $l$ :

$$
J_{M S E}=E\left[\left|A_{k} b_{j}^{(k)}(i)-\mathbf{w}_{l, j, m}^{(k) H}(i) \mathbf{r}_{l, j}^{\prime}(i)\right|^{2}\right],
$$

where $|$.$| denotes absolute value. We minimize the cost$ function (4) with respect to $\mathbf{w}_{l, j, m}^{(k) *}(i)$ and set the gradient terms equal to zero. After further mathematical manipulations [23], we have the symbol-based MMSE receivers for the $j$-th received symbol per block of user $k$

$$
\mathbf{w}_{l, j, m}^{(k)}(i)=\left(\sum_{k=1}^{K} \sum_{m=1}^{M} A_{k}^{2} \overline{\mathbf{s}}_{l, j, m}^{(k)}(i) \overline{\mathbf{s}}_{l, j, m}^{(k) H}(i)+\sigma^{2} \mathbf{I}_{N}\right)^{-1} A_{k} \overline{\mathbf{s}}_{l, j, m}^{(k)}(i),
$$

where $m=1 \ldots M$. Since the desired signal is the $j$-th symbol within this block, we should choose $m=j$ as the detector of the received data $\mathbf{r}_{l, j}^{\prime}(i)$.

2) MMSE Block-based Receiver: The block-based MMSE detector is designed as a matrix to deal with a block-length received data per time. Generally, let us consider an index $l$ $\left(l=1 \ldots 2^{B}\right)$ in the interleaver codebook, the cost function for the branch $l$ of user $k$ at the $i$-th block is given by

$$
J_{M S E}=E\left[\left\|A_{k} \mathbf{b}_{k}(i)-\mathbf{W}_{k, l}^{H}(i) \mathbf{r}_{l}(i)\right\|^{2}\right],
$$

where $\|$.$\| denotes the norm of a vector, the M N \times M$ matrix $\mathbf{W}_{k, l}(i)$ is the block-based MMSE detector for user $k$ corresponding to the branch $l$. By minimizing (6) we obtain the MMSE block-based receiver

$$
\mathbf{W}_{k, l}=\left(\sum_{k=1}^{K} A_{k}^{2} \hat{\mathbf{S}}_{k, l}(i) \hat{\mathbf{S}}_{k, l}^{H}(i)+\sigma^{2} \mathbf{I}_{M N}\right)^{-1} A_{k} \hat{\mathbf{S}}_{k, l}(i),
$$

where $\hat{\mathbf{S}}_{k, l}(i)=\mathbf{P}_{l}^{-1} \breve{\mathbf{H}}_{k}(i) \mathbf{P}_{l} \mathbf{S}_{k}$. 


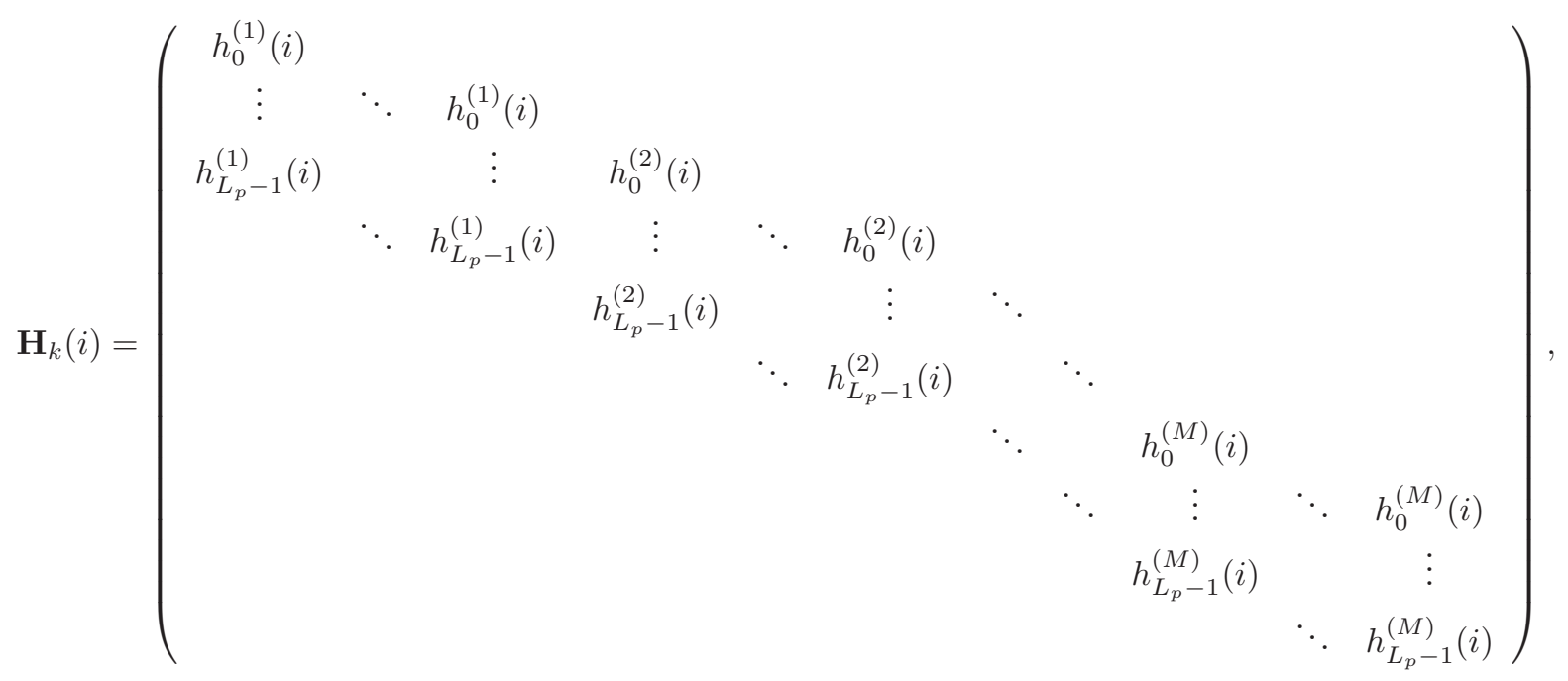

\section{System Optimization AND SElection of INTERLEAVERS}

In this section, the selection functions of the two proposed MMSE receivers for both downlink and uplink scenarios are introduced. Our proposed selection functions select the best available indices based on the channel coefficients, interleaving patterns and the spreading sequences.

\section{A. Uplink}

The system performance that we consider for the proposed uplink scheme is the sum received SINR. In this case, each user's uplink channel information is employed to compute the effective spreading sequence. The function selects the optimum index corresponding to the maximum sum received SINR over all the users in this codebook in order to feed it back to the transmitter.

1) Selection Rule for MMSE Block-based Receivers: The selection function at the base station contains all the information of chip-interleavers in this codebook, the mobile users' channels and also the MMSE receivers. The selection function uses the information to compute the received SINR of each branch for each user, and selects the optimum index to feed back to the transmitter. The received SINR of the $l$-th branch for the $k$-th user is computed as the ratio between the signals energy of user $k$ per block and the energy of interference plus noise in the same block:

$$
\begin{aligned}
\operatorname{SINR}_{l}^{(k)} & =\frac{E\left[\operatorname{Tr}\left(\mathbf{W}_{k, l}^{H}(i) \hat{\mathbf{S}}_{k, l} A_{k}^{2} \mathbf{b}_{k}(i) \mathbf{b}_{k}^{H}(i) \hat{\mathbf{S}}_{k, l}^{H} \mathbf{W}_{k, l}(i)\right)\right]}{E\left[\operatorname{Tr}\left(\mathbf{W}_{k, l}^{H}(i) \mathbf{F}_{l}(i) \mathbf{F}_{l}^{H}(i) \mathbf{W}_{k, l}(i)\right)\right]} \\
& =\frac{\operatorname{Tr}\left[\mathbf{W}_{k, l}^{H}(i) \mathbf{R}_{s}^{(l)} \mathbf{W}_{k, l}(i)\right]}{\operatorname{Tr}\left[\mathbf{W}_{k, l}^{H}(i) \mathbf{R}_{I}^{(l)} \mathbf{W}_{k, l}(i)\right]},
\end{aligned}
$$

where $\operatorname{Tr}[$.$] denotes the matrix trace calculation,$ the interference plus noise component $\mathbf{F}_{l}(i)=$ $\mathbf{r}_{l}(i)-\hat{\mathbf{S}}_{k, l} \mathbf{b}_{k}(i)$, and $M N \times M N$ matrices $\mathbf{R}_{s}^{(l)}=A_{k}^{2} \hat{\mathbf{S}}_{k, l} \hat{\mathbf{S}}_{k, l}^{H}, \quad \mathbf{R}_{I}^{(l)}=\mathbf{U}_{I, l} \mathbf{U}_{I, l}^{H}+\sigma^{2} \mathbf{I}$, $\mathbf{U}_{I, l}=\left[A_{1} \hat{\mathbf{S}}_{1, l}, \ldots, A_{k-1} \hat{\mathbf{S}}_{k-1, l}, A_{k+1} \hat{\mathbf{S}}_{k+1, l}, \ldots A_{K} \hat{\mathbf{S}}_{K, l}\right]$, and $l=1 \ldots 2^{B}, k=1 \ldots K$. The optimum index $l_{\text {opt }}$ for the uplink system maximizes the summation of the SINRs which is given by

$$
l_{\text {opt }}=\arg \max _{l=1 \ldots 2^{B}}\left\{\sum_{k=1}^{K} \operatorname{SINR}_{l}^{(k)}\right\},
$$

the final output $\hat{\mathbf{b}}_{k}^{(f)}(i)$ is given by

$$
\hat{\mathbf{b}}_{k}^{(f)}(i)=\operatorname{sgn}\left\{\Re\left(\mathbf{W}_{k, l_{o p t}}^{H}(i) \mathbf{r}_{l_{\text {opt }}}(i)\right)\right\},
$$

where $\Re($.$) selects the real part, \operatorname{sgn}\{$.$\} is the signum function,$ $\hat{\mathbf{b}}_{k}^{(f)}(i)$ is the $M \times 1$ estimation vector for the $i$-th block symbols of user $k$.

2) Selection Rule for Symbol-based Receivers: When the symbol-based receivers are employed, we assume that the $M$ symbol-based MMSE receivers $\mathbf{w}_{l, j, j}^{(k)}$ within a block for each user regarding the $l$-th branch are available for the selection function. Similarly, the received SINR within the $i$-th block is given by

$$
\operatorname{SINR}_{l}^{(k)}=\frac{\sum_{j=1}^{M} \mathbf{w}_{l, j, j}^{(k) H}(i) \mathbf{R}_{s}^{(l, k, j)} \mathbf{w}_{l, j, j}^{(k)}(i)}{\sum_{j=1}^{M} \mathbf{w}_{l, j, j}^{(k) H}(i) \mathbf{R}_{I}^{(l, k, j)} \mathbf{w}_{l, j, j}^{(k)}(i)},
$$

where the $N \times N$ matrices $\mathbf{R}_{s}^{(l, k, j)}=A_{k}^{2} \overline{\mathbf{s}}_{l, j, j}^{(k)} \overline{\mathbf{s}}_{l, j, j}^{(k) H}$, $\mathbf{R}_{I}^{(l, k, j)}=\mathbf{U}_{I, l, k, j} \mathbf{U}_{I, l, k, j}^{H}+\sigma^{2} \mathbf{I}, \quad \mathbf{U}_{I, l, k, j}=$ $\left[A_{1} \overline{\mathbf{s}}_{l, j, 1}^{(1)} \ldots A_{1} \overline{\mathbf{s}}_{l, j, M}^{(1)}, \ldots, A_{k} \overline{\mathbf{s}}_{l, j, 1}^{(k)} \ldots A_{k} \overline{\mathbf{s}}_{l, j, j-1}^{(k)} \ldots A_{k} \overline{\mathbf{s}}_{l, j, j+1}^{(k)}\right.$ $\left.\ldots A_{k} \overline{\mathbf{s}}_{l, j, M}^{(k)}, \ldots, A_{K} \overline{\mathbf{s}}_{l, j, 1}^{(K)} \ldots A_{K} \overline{\mathbf{s}}_{l, j, M}^{(K)}\right]$ denotes the interference component, namely, excludes the component $A_{k} \overline{\mathbf{s}}_{l, j, j}^{(k)}$. The selection function chooses the optimum index $l_{\text {opt }}$ corresponding to the maximum sum received SINR, and the expression is equivalent to (9). The final output of the $j$-th symbol of the $i$-th block of the $k$-th user $\hat{b}_{k}^{(f)}(i)$ is given by

$$
\hat{b}_{k}^{(f)}(i)=\operatorname{sgn}\left\{\Re\left(\mathbf{w}_{l_{o p t}, j, j}^{(k) H}(i) \mathbf{r}_{l_{o p t}, j}^{\prime}(i)\right)\right\} .
$$

\section{B. Downlink}

For the downlink, the received SINR of the user of interest is considered as the selection function. We make an assumption that the desired user knows the other users' spreading sequences, in order to obtain the theoretical received SINR 
TABLE I

COMPUTATIONAL COMPLEXITY

\begin{tabular}{cccc}
\hline Complexity & C-CDMA & \multicolumn{2}{c}{ SIDS-CDMA } \\
\hline Receivers (Block) & $O\left((M N)^{3}\right)$ & \multicolumn{2}{c}{$O\left((M N)^{3}\right)$} \\
Receivers (Symbol) & $O\left(N^{3}\right)$ & \multicolumn{2}{c}{$O\left(N^{3}+M K N^{2}\right)$} \\
\hline $\boldsymbol{\Theta}_{l}^{(k)}$ (Downlink) & - & $2(M N-1)(M N)^{2}$ & Multiplications \\
$\boldsymbol{\Theta}_{l}^{(k)}$ (Uplink) & - & $2(M N-1)(M N)^{2} K$ & $2(M N)^{3} K$ \\
$\hat{\mathbf{S}}_{k, l}$ (Block) & - & $M^{2} N(N-1) K$ & $(M N)^{2} K$ \\
$\overline{\mathbf{s}}_{l, j, m}^{(k)}$ (Symbol) & - & $M^{2} N(N-1) K$ & $(M N)^{2} K$ \\
SINR expression (Block) & - & $O\left(M^{3} N K\right)$ & $O\left(M^{3} N K\right)$ \\
SINR expression (Symbol) & - & $O\left(M^{2} N K\right)$ & $O\left(M^{2} N K\right)$ \\
\hline
\end{tabular}

expressions. Note however that the downlink SINR also can be measured and derived without the knowledge of the other spreading sequences [24] for practical use. In this case, the desired user's downlink channel information is employed to compute the effective spreading sequences instead of using all the uplink channels. The selection functions based on the block-based and symbol-based receivers can be extended in a straightforward way. For the desired user, the selection function contains the information of the interleaving codebook, the desired user's channel and the MMSE receiver.

\section{Computational Complexity}

In this part, we focus on the computational complexity of the proposed SIDS-CDMA and conventional CDMA schemes. The complexity of the matrix inversion for the block-based and symbol-based MMSE receivers are $(M N)^{3}$ and $N^{3}$ respectively [23]. In Table I, we show the complexity of the block-based and symbol-based receivers for both conventional CDMA and SIDS-CDMA systems, and the complexity of the quantities for the selection mechanisms of the proposed schemes per interleaving branch. We compute the number of additions and multiplications to compare the complexity of the equivalent channel matrix $\boldsymbol{\Theta}_{l}^{(k)}$, the effective spreading matrix $\hat{\mathbf{S}}_{k, l}$ of the block-based receiver, the effective spreading vector $\overline{\mathbf{s}}_{l, j, m}^{(k)}$ of the symbol-based receiver, and the received SINR expressions.

\section{Design of Codebooks and Low-Rate Feedback SCHEMES}

In this section, we introduce techniques to design the codebook of interleavers based on different interleaving methods. Then, the details of the low-rate feedback schemes are described and we explain how our proposed algorithms can be applied in block fading channels.

\section{A. Design of Codebooks}

The codebook design schemes like the Lloyd and Grassmannian algorithms have been widely employed in MIMO systems for beamforming and precoding techniques [10] [16]. However, they are not suitable for the interleaver-oriented limited feedback system, because the entries of the codebook are based on the permutation orders of one block of chips. The optimum interleaving codebook consists of $(M N)$ ! chipinterleavers, where (.)! represents the factorial operator, which is clearly impractical for any system. Therefore, we need to select a subset of entries from the optimum codebook to build a practical suboptimum codebook with good performance.

1) RVQ Interleaving Method: The general way to interleave the chips is to change the locations of the $M N$ chips within one block randomly, and deinterleave them at the receiver. We create the codebook by generating $2^{B}$ random interleaving patterns. Assume that the sequence $f_{1}^{(l)}, f_{2}^{(l)}, \ldots, f_{M N}^{(l)}$ is the $l$-th interleaving order in the codebook, which is a random permutation of the sequence $1,2, \ldots, M N$. The $l$-th permutation matrix is generated as follows. Firstly, the matrix $\mathbf{P}_{l}$ is initialized, we generate an $M N \times M N$ zero matrix for it. Secondly, for the $\gamma$-th row of matrix $\mathbf{P}_{l}$, the element in the $f_{\gamma}^{(l)}$-th location is changed to 1 . Thirdly, we move to the $(\gamma+1)$-th row and repeat the same approach until the element in the last row is changed, where $\gamma=1 \ldots M N$. The structure of the permutation matrix $\mathbf{P}_{l}$ is given by

$$
\mathbf{P}_{l}=\left[\mathbf{v}_{1, l}^{T}, \mathbf{v}_{2, l}^{T}, \ldots, \mathbf{v}_{M N, l}^{T}\right]^{T}
$$

where $\mathbf{v}_{\gamma, l}$ denotes the $1 \times M N$ vector, $\gamma=1 \ldots M N$,

$$
\mathbf{v}_{\gamma, l}=[\underbrace{0, \ldots, 0}_{f_{\gamma}^{(l)}-1}, 1,0, \ldots, 0] .
$$

2) Block Interleaving Method: An alternative way to permutate chips is the block interleaving method [3], which is described by (15),

$$
\boldsymbol{\Lambda}=\left(\begin{array}{ccccc}
c_{1} & c_{d+1} & c_{2 d+1} & \cdots & c_{(t-1) d+1} \\
c_{2} & c_{d+2} & c_{2 d+2} & \cdots & c_{(t-1) d+2} \\
\vdots & \vdots & \vdots & \vdots & \vdots \\
c_{d} & c_{2 d} & c_{3 d} & \cdots & c_{t d}
\end{array}\right)
$$

assuming that the dimension of the block interleaving matrix $\Lambda$ is $d$ by $t$, where $t d=M N$ is the number of chips within one block. A block of chips $c_{1}, c_{2}, \ldots, c_{t d}$ comes into the matrix $\Lambda$ column-wise, and goes out row-wise. The deinterleaving algorithm is the reverse process. By varying $t$ and $d$ we can obtain different interleaving patterns. In our experiments, for simplicity, we select the integer factors of $M N$ as the candidates of $t$ and $d$. For the $l$-th interleaver, we set $t=t^{(l)}$ and $d=d^{(l)}$. In particular, the structure of the permutation matrix $\mathbf{P}_{l}$ is given by

$$
\mathbf{P}_{l}=\left(\begin{array}{c}
\mathbf{u}_{1, l} \otimes \mathbf{I}_{t^{(l)}} \\
\mathbf{u}_{2, l} \otimes \mathbf{I}_{t^{(l)}} \\
\vdots \\
\mathbf{u}_{d^{(l)}, l} \otimes \mathbf{I}_{t^{(l)}}
\end{array}\right)
$$


where $\mathbf{u}_{\gamma, l}$ is a $1 \times d^{(l)}$ vector, $\gamma=1 \ldots d^{(l)}$,

$$
\mathbf{u}_{\gamma, l}=[\underbrace{0, \ldots, 0}_{\gamma-1}, 1,0, \ldots, 0] .
$$

3) Frequently Selected Patterns Method (FSP): The basic principle of the proposed FSP algorithm is to build a codebook which contains the interleaving patterns for the most likely selected branches. To build the codebook, we need to perform an extensive set of experiments and compute the frequency of the indices of the selected patterns. Finally, we create the codebook based on the statistics and choose the $2^{B}$ patterns, which are selected most frequently, as the entries of the codebook. The algorithm is summarized as follows.

1) Initialize the vector $\mathbf{d}_{S I N R}$, and matrices $\mathbf{L}_{i d x}$ and $\mathbf{L}_{F S P}$, generate null vector and matrices for them. $\mathbf{d}_{S I N R} \leftarrow \mathbf{0}$, $\mathbf{L}_{i d x} \leftarrow \mathbf{0}, \mathbf{L}_{F S P} \leftarrow \mathbf{0}$.

Decide the number of experiments $N_{e}$ and the length of the codebook $2^{B}$.

2) Choose an appropriate value for $\beta$, and give it to $L$.

$$
L \leftarrow \beta \text {. }
$$

3) Generate $\beta$ random interleaving patterns, give the list of the interleavers to the matrix $\mathbf{L}_{0}$.

$\mathbf{L}_{0} \leftarrow \operatorname{ordering}(\beta)$.

4) for $n_{e}=1$ to $N_{e}$ do

5) for $l=1$ to $L$ do

6) Generate the $l$-th permutation matrix corresponding to the $l$-th entry in the interleaver list $\mathbf{L}_{0}$.

$\mathbf{P}_{l} \leftarrow \mathbf{L}_{0}(l)$

7) The SINR of the $l$-th interleaver entry is computed based on the permutation matrix $\mathbf{P}_{l}$, channel matrix $\mathbf{H}_{k}$, and spreading sequences $\mathbf{s}_{k}$, give it to the $l$-th element of the vector $\mathbf{d}_{S I N R}$.

8) end

$$
\mathbf{d}_{\operatorname{SINR}}(l) \leftarrow \operatorname{SINR}\left[\mathbf{P}_{l}, \mathbf{H}_{k}, \mathbf{s}_{k}\right]
$$

9) Select the interleaver entry corresponding to the maximum SINR from the matrix $\mathbf{L}_{0}$ in the $n_{e}$-th experiment, give it to the $n_{e}$-th row of the matrix $\mathbf{L}_{i d x}$.

10) end

$$
\mathbf{L}_{i d x}\left(n_{e}\right) \leftarrow \text { MAXIndex }\left(\mathbf{d}_{\text {SINR }}\right) .
$$

11) Based on the matrix $\mathbf{L}_{i d x}$, a histogram $\operatorname{HIST}\left(\mathbf{L}_{i d x}\right)$ is generated. The codebook $\mathbf{L}_{F S P}$ is created by selecting the most frequently selected $2^{B}$ patterns according to $\operatorname{HIST}\left(\mathbf{L}_{i d x}\right)$.

$\mathbf{L}_{F S P}=\operatorname{SELECT}\left(\operatorname{HIST}\left(\mathbf{L}_{i d x}\right)\right)$,

where $\mathbf{d}_{\text {SINR }}$ denotes the vector of SINR for $\beta$ possible patterns, due to the number of all possible patterns $(M N)$ ! is dramatically large. We generate $\beta$ interleaving orders randomly, namely choose $\beta$ patterns from the optimum codebook, where $\beta$ should be a large integer and practical for the experiment. $N_{e}$ denotes the total number of experiments, $\mathbf{L}_{i d x}$ is defined for the storage of the selected patterns for every experiment. $\mathbf{L}_{0}$ is the codebook for the $\beta$ interleaving patterns generated by ordering $(\beta)$, which provides the list containing all permutations of the $\beta$ elements. We highlight that in each run, after we measure the SINR for all patterns, the pattern which brings the maximum SINR is stored in $\mathbf{L}_{i d x}$ at step 9. Finally, the FSP codebook $\mathbf{L}_{F S P}$ is created by selecting the most frequently selected $2^{B}$ patterns according to the histogram of $\mathbf{L}_{i d x}$. Note that the algorithm is described based on downlink channels. An extension scheme for the uplink is straightforward, we can follow the same approach as the downlink case. However, at step 7 the received SINR of the user of interest is replaced by the sum received SINR over all users.

\section{B. Low-rate Feedback Schemes}

For every block prior to payload transmission, there is a preamble transmission for this block channel prediction [4], [5]. The optimum index is chosen by the selection function based on the predicted one block channel information, and fed back to the transmitter before payload transmission. The feedback rate of the optimum index is once per transmission block. In particular, when the channel coefficients can be treated as constants over several blocks, we can follow the same approach to feed back the index, where the constant channel is estimated from the preamble at the receiver. The index will be only updated when the channel changes. Furthermore, error-free transmission of feedback information is not possible if the feedback channel is noisy. In the next section, we will show the performance of the novel feedback schemes based on feedback channels with errors. We will also show the performance of the novel feedback schemes based on both fading varying per symbol channels and block fading channels.

\section{Simulations}

In this section, we evaluate the performance of the novel switched interleaving schemes and compare them to other existing chip-interleaving, linear precoding and adaptive spreading algorithms. We adopt a simulation approach and conduct several experiments in order to verify the effectiveness of the proposed techniques. We carried out simulations to assess the bit error rate (BER) performance of the interleaving algorithms for different loads, signal-to-noise ratios (SNR), number of patterns and feedback errors. The users in the system are assumed to have perfect power control. All channels have a profile with 3 paths whose powers are $p_{0}=0 \mathrm{~dB}, p_{1}=-7$ $\mathrm{dB}$ and $p_{2}=-10 \mathrm{~dB}$, which are normalized, and the spacing between paths is 1 chip. The sequence of channel coefficients $h_{l}(i)=p_{l} \alpha_{l}(i)(l=0,1,2)$, where $\alpha_{l}(i)$ are zero-mean circularly symmetric complex Gaussian random variables with unit variance. We transmit 3000 blocks per frame, and a transmission block contains $M=10$ symbols. The spreading gain $N=16$ is used for the simulations. Among the different chip-interleaving algorithms and detectors, we consider:

- C-CDMA: the conventional DS-CDMA system with MMSE detector.

- G-CI-block: the general chip-interleaved algorithm of [3] exploiting the MMSE block based receiver.

- Block-CDMA: the general MMSE block-based receiver without chip-interleaving.

- MUI-free: the MUI-free algorithm proposed by Zhou et al. [8].

- MMSE-symbol: the proposed limited feedback SIDSCDMA symbol-based MMSE receiver. 


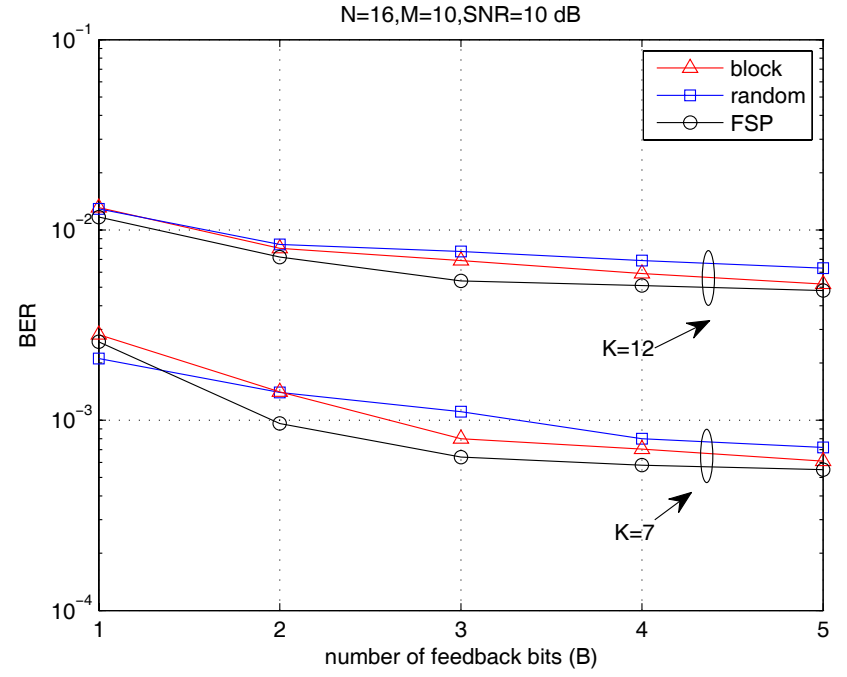

Fig. 2. BER performance versus number of feedback bits. $N=16$. Downlink system and independent multipath fading channels. $N_{e}=10000$. $\beta=1000$.

- MMSE-block: the proposed limited feedback SIDS-CDMA block-based MMSE receiver.

- $B$-bit: the limited feedback schemes employ $B$ bits, namely $2^{B}$ is the codebook length.

- precoder: the constrained transmitter precoding algorithm proposed by Vojcic and Jang [26].

- Rake: Rake receiver.

- CSI: channel state information.

- individual: the alternating update individual signature optimization algorithm [32].

- collective: the alternating update collective signature optimization algorithm [32].

In the first experiment, we compare the codebooks of the interleavers which are created by the three methods, namely the RVQ interleaving, the block interleaving and the FSP algorithms. In particular, we show BER performance curves versus number of feedback bits. Note that the codebooks are designed offline. For the FSP algorithm we set the number of simulation $N_{e}=10000$ and the number of candidates $\beta=1000$, and one block of symbols is transmitted per simulation. In the experiment which determines the FSP codebook, all the interleavers generated by the block interleaving method are employed as a part of the 1000 candidates and the rest are random interleavers. The system employs the random sequences as the spreading codes. The channel coefficients vary per symbol, which are generated independently. The perfectly predicted channel information with one block length is provided at the desired user, and the MMSE block-based receivers are used. The results for a downlink system in the scenario of multipath fading channels are illustrated in Fig. 2. We can see that the best performance is achieved with the FSP algorithm, followed by the block interleaving method and the RVQ interleaving method. The BER decreases as the number of feedback bits increases. Furthermore, the performance of the low-loaded system changes faster than the performance of the high-loaded system.
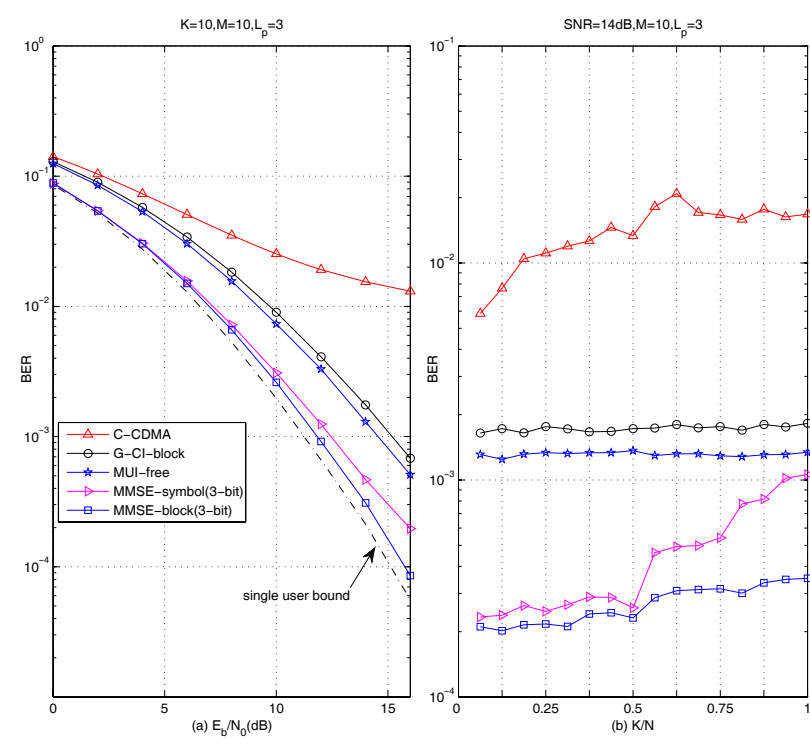

Fig. 3. BER performance versus (a) SNR and (b) $\mathrm{K} / \mathrm{N}$ for the proposed SIDSCDMA schemes, existing chip-interleaving algorithms and the conventional CDMA with MMSE receiver. Downlink block fading channels, Walsh codes and the codebook of block interleavers.

The second experiment, shown in Fig. 3, considers the comparison in terms of BER of the proposed SIDS-CDMA block-based and symbol-based MMSE receivers with the existing chip-interleaving techniques for uncoded systems in the case of downlink block fading channels. Here we use orthogonal (Walsh) codes as spreading codes, and the codebook is designed by the block interleaving approach. The channel coefficients vary every 10 blocks. For the sack of simplicity, the receivers employ perfect CSI in this experiment, the performance results with channel estimation are shown in [27]. Note that the performance hierarchy does not change with imperfect channel estimates and the performance degradation shifts the BER curves. The channel fading $\alpha_{l}(i)$ is generated independently between fading blocks. In particular, we show BER performance curves versus SNR and total number of users over processing gain $(K / N)$ for the analyzed receivers. The results in Fig. 3 indicate that the best performance is achieved with the novel SIDS-CDMA MMSE block-based receivers with 3 bits feedback ( 8 interleaving patterns), followed by the novel SIDS-CDMA MMSE symbol-based receivers with 3 bits feedback, the MUI-free algorithm proposed by Zhou et al. in [8], the general chip-interleaving algorithm [3] with blockbased MMSE receiver, and the C-CDMA MMSE receiver. Specifically, the proposed block-based MMSE receiver with 3 bits feedback can save up to almost $4 \mathrm{~dB}$ in comparison with the general chip-interleaving algorithm with block-based MMSE receiver, and can save up to over $2 \mathrm{~dB}$ in comparison with the MUI-free algorithm, at the BER level of $10^{-3}$.

In the next scenario, we compare the performance in terms of BER of the proposed SIDS-CDMA symbol-based receivers with a Rake receiver and the CDMA precoders [26] employing channel quantization techniques for downlink uncoded systems in the case of block fading channels. The systems also use Walsh codes as the spreading codes, and the channel 


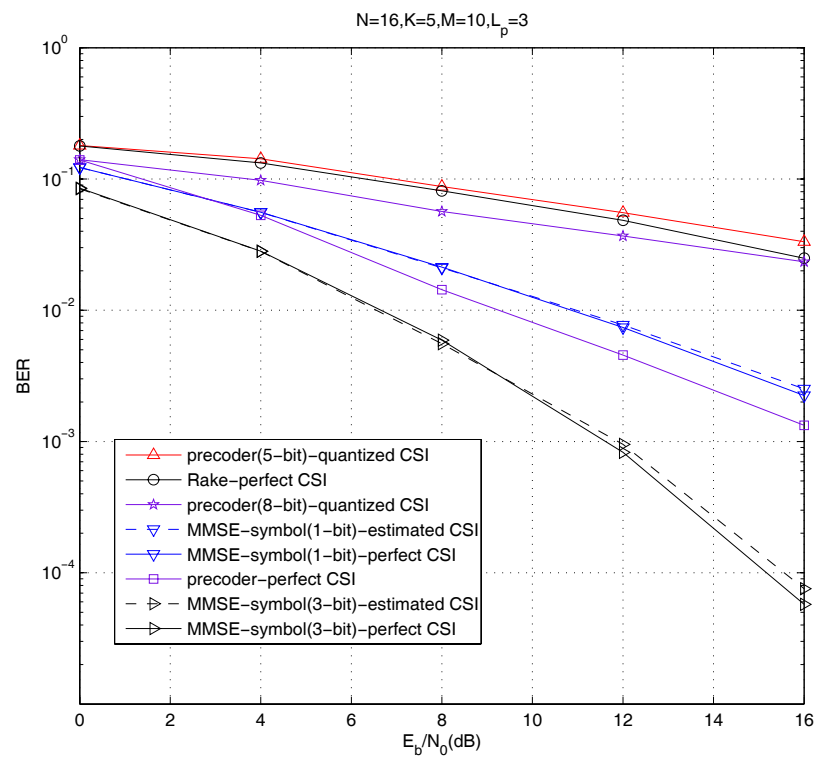

Fig. 4. BER performance versus SNR for the proposed SIDS-CDMA schemes and the conventional DS-CDMA precoders with channel quantization. Downlink block fading channels. Walsh codes and the codebook of block interleavers.

model is the same as the one in the second experiment. The bit-wise constrained transmitter precoding algorithm proposed by Vojcic and Jang [26] is considered and combined with channel quantization techniques, which works by mapping a complex valued channel vector into one of a finite number of vector realizations. The mapping is designed to minimize the distance between the input vector and the quantized vector. The codebook of the channel quantizer is designed by Grassmannian line packing algorithm [16]. From the curves in Fig. 4 we can see that the proposed symbol-based receiver based on estimated CSI approaches to a performance of that with perfect CSI. This indicates that our proposed scheme works well in a more realistic situation, where the channel estimation algorithm in [27] is employed at the receiver. The proposed switched interleaving schemes with 3 bits feedback provide the best performance. In particular, the proposed algorithm with 3 feedback bits based on estimated CSI can save up to $4 \mathrm{~dB}$ in comparison with the constrained precoder with perfect CSI at the transmitter, near the BER level of $10^{-3}$. The channel quantization technique with a relatively small number of feedback bits can not provide a good performance, due to the large quantization error and the lack of reliable CSI at the transmitter. Therefore, a significant amount of feedback bits are needed to get close to the performance of the precoder with perfect CSI [22]. The proposed scheme shows substantial performance gains with much lower requirements for feedback bits than the channel-based feedback schemes.

The results in Fig. 5(a) and Fig. 5(b) show the averaged BER performance of our proposed uplink SIDS-CDMA structure using the MMSE block based receiver with perfect and predicted CSI versus SNR and $K / N$. The systems use random sequences as the spreading codes. The channel coefficients vary per symbol in this experiment, the channel coefficients $\alpha_{l}(i)$ are computed according to Jakes' model [25], and the
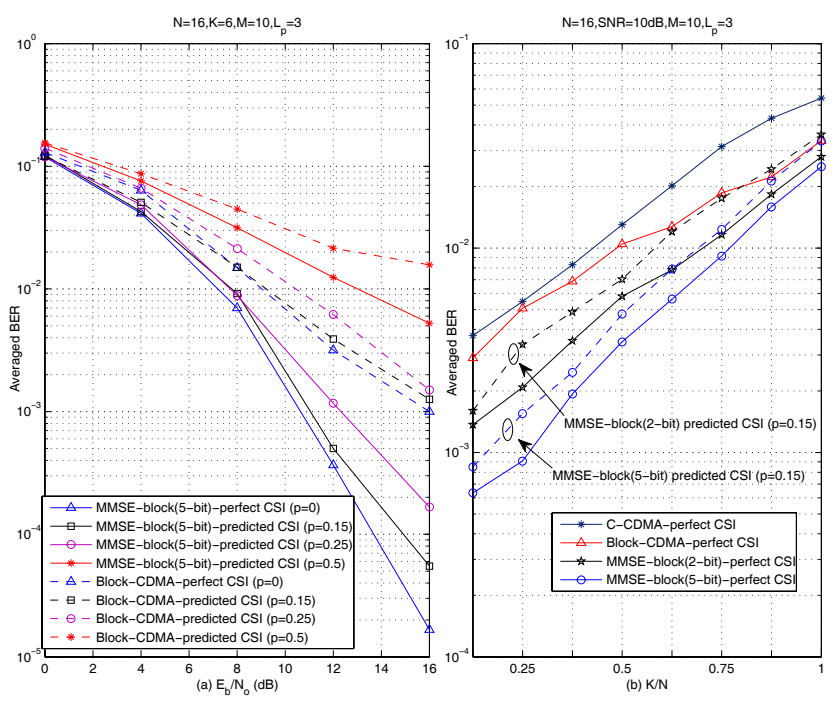

Fig. 5. Averaged BER performance versus (a) SNR and (b) K/N for the proposed SIDS-CDMA schemes, the block-based MMSE receiver and the conventional DS-CDMA with MMSE receiver. Random spreading sequence and the codebook designed by the FSP algorithm are employed. Uplink multipath fading channels with $f_{d} T=0.05$.

channel fading rate is $f_{d} T=5 \times 10^{-2}$, where the value $f_{d}$ is the Doppler shift, $f_{d}=50 \mathrm{~Hz}$, and $T$ corresponds to the block duration which contains 10 symbols, $T=10^{-3}$ seconds. This high value is a situation that represents a scenario with a high level of mobility. With lower $f_{d} T$, the prediction could be more accurate. In the simulation, we assume that the predicted channel coefficients are given by $\hat{h}_{v}^{(\beta)}(i) \approx h_{v}^{(\beta)}(i)+\varepsilon_{v}^{(\beta)}(i)$, where $\beta=1, \ldots, M, v=0, \ldots, L_{p}-1$, and $\varepsilon_{v}^{(\beta)}(i)$ denotes a complex Gaussian random variable with zero mean and variance $p^{2}$ [7]. Thus, the variance approaches the MSE of the predicted error. In practice, we refer the reader to the literature on prediction, which can be considered as an enabling technology [4], [5] and [6]. We compare the proposed limited feedback scheme with the conventional block-based MMSE receiver without limited feedback based on the quantization errors with different $p$. Here we select $p=0,0.15,0.25$ and 0.5 , and employ 5 feedback bits and 6 users. We can see that the performance decreases as the value $p$ increases, and the proposed scheme outperforms the conventional block-based MMSE receiver. In particular, the SIDS-CDMA scheme with $p=0.25$ can save up to more than $4 \mathrm{~dB}$, compared with the block-based MMSE receiver with $p=0.25$, near the BER level of $10^{-3}$. In Fig. 5(b), we compare the proposed schemes with 2 and 5 feedback bits, the block-based MMSE receivers without feedback and the C-CDMA system with MMSE receivers. We fix $S N R=10 d B$ to evaluate the averaged BER versus $K / N$. In terms of perfect CSI, the proposed SIDS-CDMA scheme with 5 feedback bits and 2 feedback bits respectively can support up to 6 and 4 more users, at the BER level of $4 \times 10^{-3}$, in comparison with the C-CDMA system with an MMSE receiver. Specifically, considering the prediction error with $p=0.15$, we can see that the SIDS-CDMA with 5 bits with predicted CSI can support up to 5 more users, at the BER level of $4 \times 10^{-3}$, 


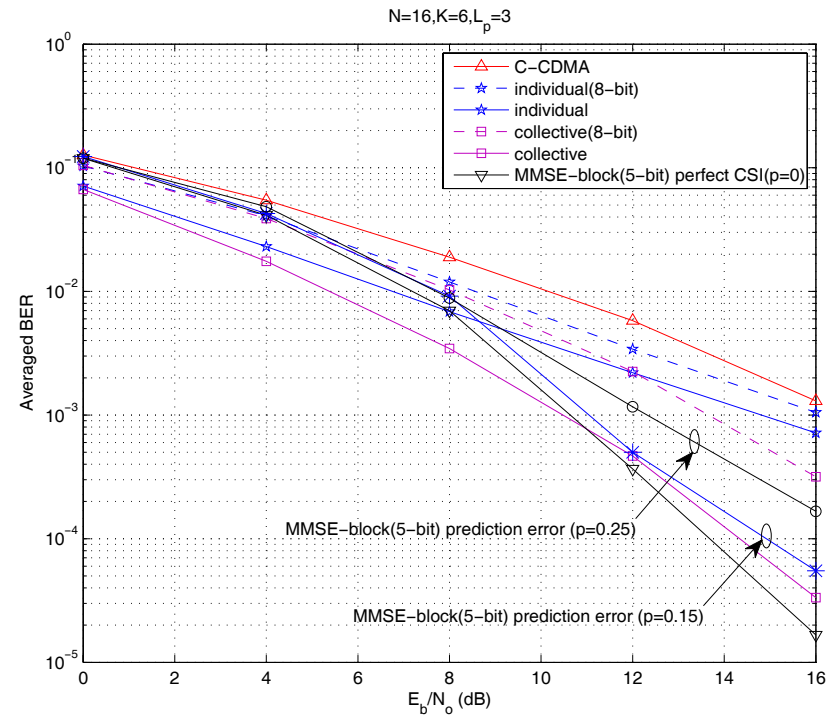

Fig. 6. Averaged BER performance versus SNR for the proposed SIDSCDMA schemes and the adaptive spreading schemes.

in comparison with the C-CDMA system with perfect CSI. Moreover, the performance advantages of the proposed limited feedback SIDS-CDMA techniques are substantially superior to the other existing approaches.

Fig. 6 shows the results of the proposed uplink scheme with the MMSE block based receiver in comparison with the results of the existing adaptive spreading techniques. In particular, we use the alternating update signature optimization algorithms proposed in [32], and combine them with the limited feedback technique. The same uplink channel scenario is used here. The Lloyd algorithm [10] is employed to generate the codebook of the quantized optimized signatures, which is isotropically distributed in an $\mathrm{N}$-dimension hypersphere of unit radius. From Fig. 6 we can see that both the individual and collective optimization algorithms are significantly better than the conventional CDMA in the case of 6 users, assuming that the optimized signature vectors are available at the transmitter. However, due to the quantization error the gains are significantly reduced. The proposed uplink scheme with predicted CSI without feedback delay using 5 feedback bits can save up to $4 \mathrm{~dB}$ compared with the individual signature optimization algorithm using 8 feedback bits and $2 \mathrm{~dB}$ compared with the collective signature optimization algorithm using 8 feedback bits, at the BER level of $10^{-3}$. Taking into account the prediction error, we can see that the proposed scheme with the channel prediction error $(p=0.25)$ can save $4 \mathrm{~dB}$ and 2 $\mathrm{dB}$, compared with the individual and the collective signature optimization algorithms using 8 feedback bits, respectively, at the BER level of $10^{-3}$.

The last two results, shown in Fig. 7 illustrate the averaged BER performance versus the percentage of each user's feedback errors for the uplink and the desired user's BER versus the percentage of the desired user's feedback errors for the downlink. We use a structure based on a frame format where the indices are converted to $0 \mathrm{~s}$ and $1 \mathrm{~s}$. This frame of $1 \mathrm{~s}$ and 0s with the feedback information is transmitted over a binary
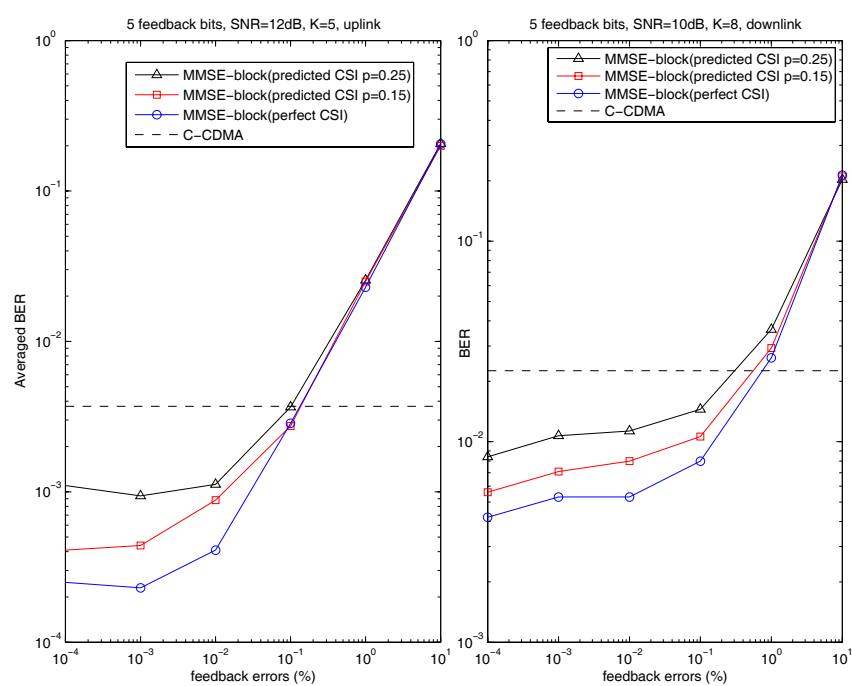

Fig. 7. BER performance versus the percentage of feedback errors for the proposed uplink and downlink schemes. The block-based MMSE receivers, random spreading sequence and the codebook designed by the FSP algorithm are employed.

symmetric channel with probability of error $P e$ associated. The burst errors scenario in the limited feedback channel can be easily transferred to the case of binary symmetric channel by employing a conventional bit interleaver. In particular, we consider to use $12 \mathrm{~dB}$ and 5 users for the uplink and $10 \mathrm{~dB}$ and 8 users for the downlink. The same fading varying per symbol channel model is employed here, and we consider to use 5 feedback bits. The random spreading sequences are employed for both the uplink and downlink. As we increase the feedback errors, the performance of the proposed limited feedback schemes decreases, since the interleaver is not in accordance with the deinterleaver due to the feedback errors. The performance of the uplink decreases fast after $0.01 \%$. In comparison with the C-CDMA, the proposed scheme with predicted CSI with $p=0.25$ starts to lose around $0.1 \%$ and $0.3 \%$ for the uplink and downlink, respectively. To ensure the errors are controlled, channel coding techniques should be employed for the feedback channels with errors.

\section{CONCLUSION}

In this paper, we proposed a novel switched interleaving technique with limited feedback for DS-CDMA system and discussed two kinds of MMSE receivers with selection functions. Three codebooks were designed by using the RVQ interleaving, block interleaving and FSP methods for the blockbased and symbol-based receivers. The results showed that the proposed interleaving and detection schemes significantly outperform existing chip-interleaving algorithms and support systems with higher loads. We remark that our proposed algorithms also can be extended to take into account coded systems, MIMO systems, OFDM systems and other types of communications systems.

\section{APPENDIX}

In this Appendix, we study the received SINR of the conventional CDMA system in comparison with the received 
SINR of the proposed SIDS-CDMA system. The received SINR of the C-CDMA system within the $i$-th block is given by

$$
\operatorname{SINR}^{(C-C D M A)}=\frac{\operatorname{Tr}\left[\boldsymbol{\Omega}_{k_{0}}^{H}(i) \overline{\mathbf{R}}_{s}(i) \boldsymbol{\Omega}_{k_{0}}(i)\right]}{\operatorname{Tr}\left[\boldsymbol{\Omega}_{k_{0}}^{H}(i) \overline{\mathbf{R}}_{I}(i) \boldsymbol{\Omega}_{k_{0}}(i)\right]}
$$

where the $M N \times M$ matrix $\boldsymbol{\Omega}_{k_{0}}(i)=$ $\operatorname{diag}\left(\left\{\boldsymbol{\omega}_{\mathrm{k}_{0}}^{(1)}(\mathrm{i}), \ldots, \boldsymbol{\omega}_{\mathrm{k}_{0}}^{(\mathrm{M})}(\mathrm{i})\right\}\right)$, the notation $\operatorname{diag}(\Phi)$ denotes the diagonal matrix whose diagonal components are taken from set $\Phi$. The vector $\boldsymbol{\omega}_{k_{0}}^{(m)}(i)$ denotes the $k_{0}$-th user 's linear MMSE receiver of the $m$-th symbol in the $i$ th block, $m=1 \ldots M$, and $\overline{\mathbf{R}}_{s}(i)=\boldsymbol{\Upsilon}_{k_{0}}(i) \boldsymbol{\Upsilon}_{k_{0}}^{H}(i)$, $\overline{\mathbf{R}}_{I}(i)=\sum_{k \neq k_{0}} \boldsymbol{\Upsilon}_{k}(i) \mathbf{\Upsilon}_{k}^{H}(i)+\sigma^{2} \mathbf{I}_{M N}$ where the $M N \times M$ matrix $\boldsymbol{\Upsilon}_{k}(i)=\operatorname{diag}\left(\left\{\boldsymbol{v}_{\mathrm{k}, 1}(\mathrm{i}), \ldots, \boldsymbol{v}_{\mathrm{k}, \mathrm{M}}(\mathrm{i})\right\}\right)$. Comparing (8) with (18), note that the received SINR of the conventional CDMA system is a particular branch of the proposed SIDSCDMA system, which corresponds to the scheme without chip-interleaving, namely, the permutation matrix $\mathbf{P}_{l}=\mathbf{I}_{M N}$. The proposed algorithm selects the optimum interleaver for the $i$-th block, which can maximize the received SINR over the block. Thus, when there is no chip-interleaving applied to the transmitted signal, we obtain the inequation as follows,

$$
\operatorname{SINR}^{(C-C D M A)} \leq \operatorname{SINR}^{(S I D S-C D M A)} .
$$

This analytically establishes that the proposed limited feedback approach outperforms the conventional DS-CDMA system with MMSE receiver, and shows the effect of switched interleaving on the SINR.

\section{REFERENCES}

[1] G. D. Forney, Jr., "Burst-correcting codes for the classic bursty channel," IEEE Trans. Commun., vol. 19, no. 5, pp. 772-781, Oct. 1971.

[2] Y. N. Lin and D. W. Lin, "Multiple access over fading multipath channels employing chip-interleaving code-division direct-sequence spread spectrum," IEICE Trans. Commun., vol. E86-B, no. 1, pp. 114-121, Jan. 2003.

[3] Y. Na, M. Saquib, and M. Z. Win, "Pilot-aided chip-interleaved DSCDMA transmission over time-varying channels," IEEE J. Sel. Areas Commun., vol. 24, no. 1, pp. 151-160, Jan. 2006.

[4] A. Duel-Hallen, S. Hu, and H. Hallen, "Long-range prediction of fading signals," IEEE Signal Process. Mag., vol. 15, no. 3, pp. 62-75, May 2000.

[5] A. Duel-Hallen, "Fading channel prediction for mobile radio adaptive transmission systems," Proc. IEEE, vol. 95, no. 12, pp. 2299-2313, Dec. 2007.

[6] J. Heo, Y. Wang, and K. Chang, "A novel two-step channel-prediction technique for supporting adaptive transmission in OFDM/FDD system," IEEE Trans. Veh. Technol., vol. 57, no. 1, pp. 188-193. Jan. 2008.

[7] N. S. Jayant and P. Noll, Digital Coding of Waveforms: Principles and Applications to Speech and Video. Prentice-Hall, 1984.

[8] S. Zhou, G. B. Giannakis, and C. Martret, "Chip-interleaved blockspread code division multiple access," IEEE Trans. Commun., vol. 50, no. 2, pp. 235-248, Feb. 2002.

[9] C. Schlegel, "CDMA with partitioned spreading," IEEE Commun. Lett., vol. 11, no. 12, pp. 913-915, Dec. 2007.

[10] A. Narula, M. J. Lopez, M. D. Trott, and G. W. Wornell, "Efficient use of side information in multiple-antenna data transmission over fading channels," IEEE J. Sel. Areas Commun., vol. 16, no. 8, pp. 1423-1436, Oct. 1998.

[11] B. C. Banister and J. R. Zeidler, "Feedback assisted transmission subspace tracking for MIMO systems," IEEE J. Sel. Areas Commun., vol. 21, no. 3, pp. 452-463, Apr. 2003.

[12] J. C. Roh and B. D. Rao, "Multiple antenna channels with partial channel state information at the transmitter," IEEE Trans. Wireless Commun., vol. 3, no. 2, pp. 677-688, Mar. 2004.

[13] M. Sharif and B. Hassibi, "On the capacity of MIMO broadcast channels with partial side information," IEEE Trans. Inf. Theory, vol. 51, no. 2, Feb. 2005.
[14] D. J. Love, R. W. Heath, Jr., V. K. N. Lau, D. Gesbert, B. D. Rao, and M. Andrews, "An overview of limited feedback in wireless communication systems," IEEE J. Sel. Areas Commun., vol. 26, no. 8, pp. 1341-1365, Oct. 2008.

[15] D. J. Love and R. W. Heath, Jr., "Limited feedback unitary precoding for orthogonal space-time block codes," IEEE Trans. Signal Process., vol. 53, no. 1, pp. 64-73, Jan. 2005.

[16] D. J. Love, R. W. Heath, Jr., and T. Strohmer, "Grassmannian beamforming for multiple-input multiple-output wireless systems," IEEE Trans. Inf. Theory, vol. 49, no. 10, pp. 2735-45, Oct. 2003.

[17] K. K. Mukkavilli et al., "On beamforming with finite rate feedback in multiple-antenna systems," IEEE Trans. Inf. Theory, vol. 49, no. 10, pp. 2562-79, Oct. 2003.

[18] D. J. Love and R. W. Heath, Jr., "Grassmannian precoding for spatial multiplexing systems," in Proc. Allerton Conf. Commun., Control, Comput., Oct. 2003.

[19] P. Ding, D. J. Love, and M. D. Zoltowski, "Multiple antenna broadcast channels with shape feedback and limited feedback," IEEE Trans. Signal Process., vol. 55, no. 4, July 2007, pp. 3417-3428.

[20] A. D. Dabbagh and D. J. Love, "Multiple antenna MMSE based downlink precoding with quantized feedback or channel mismatch," IEEE Trans. Commun., vol. 56, no. 11, pp. 1859-1868, Nov. 2008.

[21] R. S. Blum, "MIMO with limited feedback of channel state information," in Proc. ICASSP, vol. 4, pp. 89-92, Apr. 2003.

[22] D. J. Love, R. W. Heath, Jr., W. Santipach, and M. L. Honig, "What is the value of limited feedback for MIMO channels?" IEEE Commun. Mag., vol. 42, no. 10, pp. 54-59, Oct. 2004.

[23] S. Verdu, Multiuser Detection. Cambridge, 1998.

[24] S. Moshavi, D. Yellin, J. S. Sadowsky, Y. Perets, and K. Pick, "Pilot interference cancellation technology for CDMA cellular networks," IEEE Trans. Veh. Technol. vol. 54, no. 5, pp. 1781-1792, Sep. 2005.

[25] A. Goldsmith, Wireless Communications. Cambridge University Press, 2005.

[26] B. R. Vojcic and W. M. Jang, "Transmitter precoding in synchronous multiuser communications," IEEE Trans. Commun., vol. 46, no. 10, pp. 1346-1355, Oct. 1998.

[27] Y. Cai, R. C. de Lamare, and R. Fa, "Linear interference suppression for spread spectrum systems with switched interleaving and limited feedback," in Proc. IEEE Wireless Commun. Netw. Conf., Apr. 2009.

[28] Y. Na and M. Saquib, "Effects of time-variations in wireless channels on the two different fade-resistant CDMA systems," IEEE Trans. Wireless Commun., vol. 7, no. 8, pp. 2923-2929, Aug. 2008.

[29] C. Rose, S. Ulukus, and R. Yates, "Wireless systems and interference avoidance," IEEE Trans. Wireless Commun., vol. 1, no. 3, pp. 415-428, July 2002

[30] S. Ulukus and R. Yates, "Iterative construction of optimum signature sequence sets in synchronous CDMA systems," IEEE Trans. Inf. Theory, vol. 47, no. 5, pp. 1989-1998, July 2001.

[31] C. Rose, "CDMA codeword optimization: interference avoidance and convergence via class warfare," IEEE Trans. Inf. Theory, vol. 47, no. 6, pp. 2368-2382, Sep. 2001.

[32] G. S. Rajappan and M. L. Honig, "Signature sequence adaptation for DS-CDMA with multipath," IEEE J. Sel. Areas Commun., vol. 20, no. 2, pp. 384-395, Feb. 2002.

[33] W. Santipach and M. L. Honig, "Signature optimization for CDMA with limited feedback," IEEE Trans. Inf. Theory, vol. 51, no. 10, pp. 3475-3492, Oct. 2005.

[34] G. Woodward, R. Ratasuk, M. L. Honig, and P. B. Rapajic, "Minimum mean-squared error multiuser decision-feedback detectors for DSCDMA," IEEE Trans. Commun., vol. 50, no. 12, pp. 2104-2112, Dec. 2002.

[35] R. C. de Lamare and R. Sampaio-Neto, "Minimum mean-squared error iterative successive parallel arbitrated decision feedback detectors for DS-CDMA systems," IEEE Trans. Commun., vol. 56, no. 5, pp. 778789, May 2008

[36] R. C. de Lamare, R. Sampaio-Neto, and A. Hjorungnes, "Joint iterative interference cancellation and parameter estimation for CDMA systems," IEEE Commun. Lett., vol. 11, no. 12, pp. 916-918, Dec. 2007. 


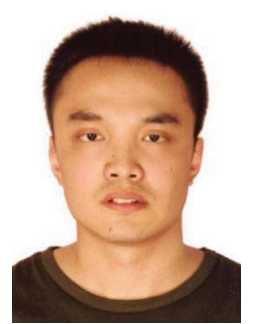

Yunlong Cai (S'07 - M'10) received the B.S. degree in computer science from Beijing Jiaotong University, Beijing, China, in 2004, the M.Sc. degree from the University of Surrey, Guildford, U.K. in 2006, and the Ph.D. degree from the University of York, York, U.K., in 2009, both in electronic engineering. From January 2010 to January 2011, he was a Post-Doctoral Fellow at the Electronics and Communications Laboratory of the Conservatoire National des Arts et Métiers (CNAM), Paris, France. Since February 2011, he has been with the Department of Information Science and Electronic Engineering at Zhejiang University, Hangzhou, China, where he is currently a Lecturer. His research interests include spread spectrum communications, adaptive signal processing, multiuser detection, and multiple antenna systems.

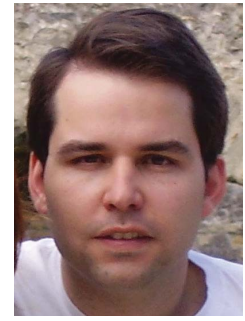

Rodrigo C. de Lamare (S'99 - M'04 - SM'10) received the Diploma in electronic engineering from the Federal University of Rio de Janeiro (UFRJ) in 1998 and the M.Sc. and PhD degrees, both in electrical engineering, from the Pontifical Catholic University of Rio de Janeiro (PUC-Rio) in 2001 and 2004, respectively. Since January 2006, he has been with the Communications Research Group, Department of Electronics, University of York, where he is currently a lecturer in communications engineering. His research interests lie in communications and signal processing, areas in which he has published about 200 papers in refereed journals and conferences. Dr. de Lamare serves as associate editor for the EURASIP Journal on Wireless Communications and Networking. He is a Senior Member of the IEEE and has served as the General Chair of the 7th IEEE International Symposium on Wireless Communications Systems, held in York, UK in September 2010.

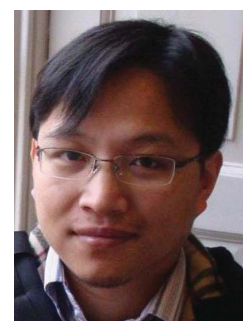

Rui Fa received his Bachelor and Master degrees in electronic and electrical engineering from Nanjing University of Science and Technology (NUST), China, in 2000 and in 2003, respectively, and his $\mathrm{PhD}$ degree in electrical engineering from University of Newcastle, UK, in 2007. From January 2008 to February 2010, he was with the University of York, where he was a postdoctoral research associate. From March 2010 to October 2010, he was a research associate with the University of Leeds. Since November 2010, he has been with the University of Liverpool. His current research interests include radar signal processing, signal processing and information theory for wireless communications, data and signal processing for biotechnology. 